\title{
La metodología de control emocional en conversaciones con padres, madres y personas apoderadas y el desarrollo de habilidades conversacionales en docentes. Evidencia desde Chile
}

\author{
The Methodology of Emotional Control in Conversations With Parents and the Development \\ of Conversational Skills in Teachers. Evidence From Chile
}

\begin{abstract}
Metodologia do controle emocional para conversas com os pais, mães e pessoas apoderadas e o desenvolvimento de habilidades comunicativas para professores: Evidências de Chile
\end{abstract}

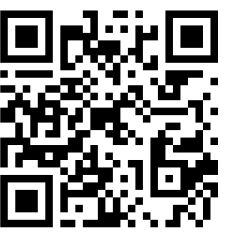

Marta Quiroga-Lobos Pontificia Universidad Católica de Valparaíso Valparíso, Chile marta.quiroga@pucv.cl iD https://orcid.org/0000-0003-3630-384X

Felipe Aravena-Castillo Pontificia Universidad Católica de Valparaíso Valparíso, Chile felipe.aravena@pucv.cl https://orcid.org/0000-0002-9142-8349

Recibido • Received • Recebido: 20 / 10 / 2018

Corregido • Revised • Revisado: 06 / 03 / 2020

Aceptado • Accepted • Aprovado: 21/ 07 / 2020

\begin{abstract}
Resumen: El presente estudio explica y evalúa la metodología CECA (control emocional en conversaciones con apoderados) a través de su implementación en una escuela primaria en Chile. El objetivo de la metodología es desarrollar habilidades en el profesorado jefe para realizar entrevistas con personas adultas clasificadas como difíciles, y que son padres y madres de estudiantes con bajos resultados académicos y con conductas disruptivas en el aula. Paralelo a la formación, se aplicó el modelo de evaluación de D. L. Kirkpatrick y J. D. Kirkpatrick (2006) en sus cuatro pasos: satisfacción, adquisición de conocimientos teóricos, sustentabilidad de las prácticas y efectos organizacionales. Se concluyó que la metodología desarrolla en el profesorado habilidades para reconocer emociones, seleccionar tipos de conversaciones, analizar evidencias de aprendizaje con padres, madres y personas apoderadas, y planificar, en conjunto, metas de corto plazo asociadas al aprendizaje. Los resultados enriquecen la propuesta metodológica en tres aspectos: el primero, hacer los ciclos
\end{abstract}


http://doi.org/10.15359/ree.24-3.10

http://www.una.ac.cr/educare

educare@una.ac.cr

de retroalimentación intensivos inicialmente y que se difieran paulatinamente, acorde con las necesidades de las personas participantes; el segundo, incluir en la metodología acciones específicas para el equipo directivo sobre gestión del tiempo, gestión de infraestructura y animación del trabajo colaborativo entre pares; y el tercero, que esta metodología puede ser transferida a otros contextos formativos, ya que satisface necesidades reales en el sistema escolar.

Palabras clave: Profesorado jefe; conversaciones desafiantes; emociones; retroalimentación; personas apoderadas.

Abstract: The present study explains and evaluates the ECSC methodology (Emotional Control in Conversations with Parents) through its implementation in a primary school in Chile. The methodology's goal is to develop skills in the teachers' heads to conduct interviews with parents classified as difficult, and parents of students with low academic results and/or disruptive behavior in the classroom. Parallel to training, the D. L. Kirkpatrick and J. D. Kirkpatrick (2006) evaluation model was applied in its four steps: satisfaction, acquisition of theoretical knowledge, the sustainability of practices, and organizational effects. It was concluded that the methodology could develop skills for teachers to recognize emotions, select types of conversations, analyze evidence of learning with parents and plan, together, short-term goals associated with learning. The results also enrich the methodological proposal in three aspects: first, to make the intensive feedback cycles initially and to gradually differ according to the needs of the participants; second, to include specific actions in the methodology, for the management team, on time management, infrastructure management, and the animation of collaborative work among peers; third, this methodology can be transferred to other formative contexts, since it satisfies real needs in the school system.

Keywords: Teacher; challenging conversations; emotions; feedback; parents.

Resumo: Este estudo explica e avalia a metodologia CECA (Controle Emocional em Conversas com Apoderados) através da sua implementação em uma escola primária no Chile. O objetivo da metodologia é desenvolver habilidades nos professores para realizar entrevistas com pessoas adultas classificados como difíceis, e que são pais e mães de estudantes com baixos resultados acadêmicos e / ou com comportamento irruptivo na sala de aula. Paralelamente ao treinamento, aplicou-se o modelo de avaliação de D. L. Kirkpatrick e J. D. Kirkpatrick (2006) em suas quatro etapas: satisfação, aquisição de conhecimento teórico, sustentabilidade das práticas e efeitos organizacionais. Concluiu-se que a metodologia desenvolve nos professores habilidades para reconhecer emoções, selecionar tipos de conversas, analisar evidências de aprendizagem com pais e mães e planejar conjuntamente metas de curto prazo associadas à aprendizagem. Os resultados enriquecem a proposta metodológica em três aspectos: o primeiro, fazer ciclos intensivos de retroalimentação inicialmente e que vai mudando gradualmente de acordo com as necessidades dos participantes; o segundo, incluir na metodologia ações específicas para a equipe de direção sobre o gerenciamento do tempo, da infraestrutura e animação de trabalho colaborativo entre duplas; e o terceiro, que esta metodologia pode ser transferida para outros contextos de formação, uma vez que satisfaz as necessidades reais do sistema escolar.

Palavras chave: professores chefes; conversas desafiadoras; emoções; retroalimentação; representantes. 


\section{Introducción}

Las escuelas chilenas adscritas a la ley de Subvención Escolar Preferencial (SEP) cuentan con recursos financieros adicionales que permiten contratar servicios de asistencia técnica. Utilizando esta facultad, el equipo directivo de una escuela básica que atiende a alumnos y alumnas vulnerables, solicitó a tres profesionales formar a su profesorado en puestos de jefatura $(n=14)$ y equipo directivo $(n=4)$ en la metodología control emocional en conversaciones con apoderados (CECA). Esta metodología busca desarrollar habilidades conversacionales para realizar entrevistas con personas apoderadas clasificadas como difíciles por el profesorado jefe, y que son padres y madres de estudiantes con actitudes disruptivas y bajos resultados de aprendizaje.

Esta necesidad nace del diagnóstico realizado por el equipo directivo, que evidencia los siguientes problemas: a) Los sujetos directivos ocupan un tiempo excesivo en atender a personas apoderadas. b) El profesorado jefe deriva frecuentemente a las personas apoderadas difíciles para que sean atendidas por algún o alguna integrante del equipo directivo. c) El equipo directivo considera que muchas situaciones se podrían solucionar anticipadamente, si el profesorado jefe mantuviera una comunicación más frecuente con los sujetos apoderados, y d) El profesorado jefe manifiesta que en algunas oportunidades sufren violencia verbal de los sujetos apoderados, por eso los derivan para que sean entrevistados por personal directivo docente.

A partir de este diagnóstico, se propone, al equipo directivo, la formación del profesorado en la metodología CECA, proceso formativo que fue evaluado utilizando el modelo de cuatro niveles de D. L. Kirkpatrick y J. D. Kirkpatrick (2006).

\section{Relación familia-escuela: Un binomio necesario para potenciar los aprendizajes}

La relación positiva entre la escuela y la familia favorece aprendizajes de calidad en estudiantes. Esto ha sido documentado en diversas investigaciones (Brunner y Elacqua, 2003; Casassus et al., 2001; CIDE y UNICEF, 2000; Epstein, 2001; Eurydice, 1997; Henderson et al., 2007; Martiniello, 1999; U. S. Department of Education, 1994). También se ha encontrado evidencia de que el desarrollo curricular, centrado en las intersecciones de los niños y las niñas, las familias y las comunidades, contribuye al aprendizaje (Clandinin y Connelly, 1992; Clandinin et al., 2006; Epstein, 2001). Diversos estudios sobre lectura (Domina, 2005; Fan y Williams, 2010; Jeynes, 2005; Jeynes, 2012; Powell-Smith et al., 2000) y matemáticas (Sheldon y Epstein, 2005; Sirvani, 2007) han llegado a la misma conclusión. Asimismo, se ha encontrado una asociación positiva entre la asistencia a clases y la preparación de clase, cuando la relación padres, madres y escuela es positiva (Simon, 2001), y negativa cuando existen problemas de comportamiento del estudiantado (Domina, 2005) y hay altas tasas de deserción (Barnard, 2004). 
Para el fortalecimiento de la relación escuela-familia, Henderson et al. (2007) proponen que los programas de participación de la familia estén orientados a tratar temas asociados con el bienestar estudiantil, resultados académicos y brindar apoyos permanentes a las familias. Estos temas son especialmente valiosos para los sujetos apoderados de grupos vulnerables o marginados en las escuelas (Pushor, 2007; Ramírez, 2003). Esto contribuye a superar estereotipos de clase (Anyon, 1980), por lo que los programas de apoyo emocional son una valiosa herramienta para la familia, ya que ésta es la primera maestra de su hijo o hija (Bridges et al., 2012).

La participación de las familias en la escuela es necesaria, ya que ellas tienen un conjunto de recursos y conocimientos vitales, lingüísticos, históricos, políticos y culturales que explican su posición y la del estudiantado en el mundo (Yosso, 2005). La importancia de la participación de las familias en las escuelas se ha manifestado en diversas iniciativas de políticas públicas, como por ejemplo, la ley No Child Left Behind de USA, la conformación en Noruega del Comité de Padres y, en el caso chileno, la creación de los Consejos Escolares. El estudio de Dorthe Tveit (2009), realizado en Noruega, analizó las actas de reuniones del Comité Nacional de Padres para la Educación Primaria y Secundaria, y concluyó que los padres y madres, pese a tener derechos legales, deben constantemente legitimarlos. El estudio etnográfico realizado por Bergnehr (2015) sobre la política de apoyo a padres y madres en Suiza, concluye que, pese a que la política establece la creación de equipos interdisciplinarios para apoyar a los padres y madres, la relación con estos grupos la realiza fundamentalmente el profesorado, con una baja utilización de otros equipos profesionales. De lo anterior se concluye que la colaboración escuela-familia aún requiere de más investigación y del desarrollo de programas que incentiven la participación.

\section{Rol del profesorado jefe}

En Chile, el vínculo entre familia y escuela se materializa en la persona apoderada (miembro del grupo familiar o tutora del alumnado) y profesorado jefe. Tradicionalmente, él o ella están a cargo de un grupo de estudiantes que conforman un grupo curso, que en promedio varía entre 20 y 40 estudiantes, e imparte alguna asignatura del currículo. El rol de profesorado jefe nace en el año 1927 y originalmente respondió a las exigencias de las organizaciones docentes (Collao et al., 1998). Junto al rol se creó la asignatura de Consejo de curso, que inicialmente se orientó a estimular la participación democrática del alumnado. Durante la época de la dictadura (1973 a 1990), la asignatura fue reorientada a tres tipos de tareas: una, asociada a estudiantes, que implicaba apoyo individual sobre rendimiento académico, orientación vocacional y solución de problemas de comportamiento disruptivo; otra, asociada a los sujetos apoderados, que requería la realización de reuniones mensuales con el grupo de apoderados del curso y de entrevistas individuales; y la última, asociada a tareas administrativas, que consistía en la confección de documentación, lo que despolitiza su función (Ramírez Muga, 2015). Este artículo se focaliza específicamente en las conversaciones individuales con personas apoderadas definidas como difíciles por el profesorado jefe.

4 Marta Quiroga-Lobos y Felipe Aravena-Castillo

Los artículos de la Revista Electrónica Educare del Centro de Investigación y Docencia en Educación de la Universidad Nacional, Costa Rica, se comparten bajo términos de la Licencia Creative Commons: Reconocimiento, № Comercial, Sin Obra Derivada 3.0 Costa Rica. Las autorizaciones adicionales a las aquí delimitadas se pueden obtener en el correo: educare@una.cr 


\section{Relación padres, madres, sujetos apoderados y profesorado}

Padres, madres y profesorado tienen diferentes perspectivas sobre el proceso educativo (Casanova, 1996; de Carvalho, 2001; Lawson, 2003; Waggoner y Griffith, 1998). La formación socioemocional de profesorado jefe se asocia positivamente con la mejora de la autoestima de estudiantes y el rendimiento académico. Estos hallazgos se documentan en el estudio de Marchant Orrego et al. (2013), realizado en dos colegios que atienden a alumnado vulnerable. Estos resultados coinciden con lo planteado por Hargreaves $(2001 a, b)$, respecto a que las emociones positivas como la satisfacción y el compromiso son muy importantes para el profesorado, y que las críticas de los padres y madres provocan sentimientos de impotencia en este grupo. El estudio de Villarroel (2011) muestra "una asociación lineal entre autoconcepto y rendimiento académico, la influencia recíproca entre las expectativas del profesor jefe, el autoconcepto y rendimiento del alumno, y el efecto que tiene el rendimiento logrado por el alumno sobre la percepción que el profesor tiene de él" (p. 3). También se han creado abundantes manuales para guiar a profesorado jefe, pero no se han encontrado experiencias documentadas sobre el desarrollo de habilidades de conversación del profesorado jefe. Por ello, se hace necesario explorar en técnicas específicas que ayuden a profesorado jefe a gestionar conversaciones difíciles.

\section{Metodología CECA (control emocional en conversaciones con apoderados)}

Desde la perspectiva de Le Fevre y Robinson (2014), las conversaciones difíciles se producen a partir de una "queja" que se comunica al sujeto apoderado en general sobre situaciones disruptivas o bajos resultados de aprendizaje de sus hijos o hijas. Estas conversaciones son desafiantes para el profesorado y el sujeto apoderado, porque tienen un impacto emocional en ambos; no se pueden anticipar los resultados y demandan el desarrollo de acciones compartidas. A continuación, se presentan los referentes teóricos que sustentan la metodología CECA.

a) Conductas disruptivas en el aula: Estas fueron analizadas a partir de la perspectiva de teoría del apego y su influencia en el aprendizaje y en las actitudes sociales de estudiantes (Bowlby, 1998; Fonagy et al., 2002; Geddes, 2006; Hughes y Schlösser, 2014), Marrone, 2001; Pinedo Palacios y Santelices Álvarez, 2006); especialmente útiles en niñez del primer ciclo básico. También, se analizaron las implicancias de utilizar principios conductistas, recompensas y sanciones en el aula para mantener el orden (Wong et al., 2012), y cómo el profesorado que utiliza estrategias reactivas (es decir, como respuesta a una mala conducta después de que esta ha ocurrido), tiende a sentirse más estresado que quienes utilizan estrategias proactivas (que previenen los problemas de conducta antes de que se produzcan), como se reporta en el estudio de Clunies-Ross et al. (2008). Todo ello, con el objetivo de potenciar la comprensión de la historia familiar (apego) y cómo las actitudes de docentes deben conjugarse para lograr las condiciones propicias para el aprendizaje. 
b) Conversaciones y emociones: Se combinaron tres líneas teóricas: Bloch (2008), Echeverría (2007) y Hargreaves (2001a). La primera permite a maestros y maestras comprender sus emociones; la segunda, identificar los tipos de conversaciones y la tercera conceptualizar los tipos de distancia emocional. Es decir, se interrelacionan razones socioculturales que explican la distancia emocional entre profesorado y sujetos apoderados con las expresiones corporales, fisiológicas y subjetivas de las emociones, lo que le permite, al profesorado jefe, configurar un diagnóstico y seleccionar el tipo de conversación más adecuado. A continuación, se presenta una breve síntesis de estas tres líneas teóricas:

b1) Geografía emocional: Hargreaves (2001a), identificó cinco dimensiones emocionales: (1) distancia sociocultural, donde las diferencias etnoculturales y de clase entre maestros, maestras, madres, padres o sujetos tutores les hacen sentirse como grupos ajenos e incognoscibles entre sí. Específicamente, el personal docente a menudo se ven a sí mismo enseñando a los "hijos de otras personas" y también consideran a las madres y padres obreros como la "alteridad" (Delpit, 1993; Lindblad y Pérez Prieto, 1992). (2) Distancia moral, la que ocurre, por ejemplo, cuando los maestros o maestras sienten que sus propósitos están siendo amenazados por la crítica de los padres, madres; o apoyados cuando son reconocidos por los padres (Hargreaves, 2001b). (3) Distancia profesional, la que consiste en la profesionalidad del profesorado, definida como un modelo clásico basado en los principios tradicionalmente masculinos que deben evitar enredos emocionales con sus clientes (es decir, los padres y madres) y mantener una distancia profesional (Grumet, 1988; Hargreaves y Goodson, 1996). (4) Distancia física: las interacciones entre profesorado, y padres y madres suelen ser infrecuentes, fragmentadas y emergentes a partir de disrupciones en clases o bajos resultados, lo que dificulta un diálogo permanente (Lasky, 2000). (5) Distancia política: las relaciones de poder jerárquicas pueden distorsionar los aspectos emocionales de la comunicación entre docentes y quienes les rodean (Blase y Anderson, 1995).

b2) Emociones: Bloch (2008) desarrolló el sistema Alba Emoting que permite al "usuario entrenado, expresar y modular mensajes emocionales precisos que logren comunicar correctamente lo deseado, sin distorsiones ni equívocos, y en forma rápida y eficiente" (p. 114). El modelo diferencia seis emociones básicas (alegría, rabia, miedo, pena, erotismo y ternura). Para cada una de ellas se han identificado tres niveles que actúan interconectadamente: "un nivel expresivo (facial/postural), un nivel fisiológico (orgánico) y un nivel subjetivo (mental)" (p. 51). El sistema determina una configuración respiratoria y somática expresiva 
particular para cada emoción básica, Ilamada"[factor] emocional, a partir de la cual cada emoción puede ser reconocible, si observamos los parámetros indicados" (p. 155). El entrenamiento permite al público usuario conectarse y reconocer en otros sujetos los estados emocionales básicos y mixtos (combinación de básicos). Un aspecto fundamental de esta técnica es el estado de neutralidad, que se caracteriza por un ritmo calmado y regular de respiración y una expresión facial neutra. Para lograr este estado, el sistema desarrolló un paso denominado Step out, que permite salirse de la emoción básica o mixta y lograr un estado de serenidad. Este es un elemento fundamental para iniciar y sostener conversaciones desafiantes y comprender el estado emocional del sujeto apoderado.

b3) Tipología de conversaciones: Echeverría (2007) analiza el rol del lenguaje en las conversaciones, las razones de los quiebres y su vínculo con las tipologías de conversaciones. Especifica cuatro tipos de conversaciones que pueden ser utilizadas en diferentes situaciones, y que se relacionan con el tipo de quiebre. Los cuatro tipos de conversaciones son: a) Conversaciones de juicios personales. En ellas normalmente se reconstruye el quiebre y los significados que cada hablante asocia a los eventos. Son conversaciones descriptivas y de juicios personales sobre la situación. b) Conversaciones para coordinar acciones. Se caracterizan porque acuerdan acciones futuras para cambiar el curso actual de las cosas, lo cual produce el quiebre. Si tiene éxito, normalmente se puede esperar que el quiebre sea superado. Este tipo de conversaciones es frecuente cuando de ambas partes existe acuerdo o intensión de mejorar. c) Conversaciones para posibles acciones. Se realizan cuando se juzga que no se puede sostener una determinada conversación con alguien, por lo que es difícil acordar acciones y quizás resulte más apropiado realizar una conversación de juicios personales, y d) Conversaciones para posibles conversaciones. Son apropiadas para identificar oportunidades de conversación. No se logra compartir juicios personales ni acordar posibles acciones. La elección del tipo de conversaciones depende del efecto emocional del quiebre.

Interrelacionar las perspectivas de Bloch (2008), Echeverría (2007) y Hargreaves (2001a) permite al profesorado jefe comprender la naturaleza de la distancia emocional que lo separa de sus sujetos apoderados, las emociones que predominan y el tipo de conversación que pueden realizar. Estos conocimientos ayudan a tomar decisiones fundamentadas y a anticipar efectos deseados y no deseados, especialmente en sus emociones. En definitiva, admite conocer más su propia persona y a comprender las diversas barreras que tienen para sostener conversaciones desafiantes con sus sujetos apoderados. 


\section{Proceso formativo}

Se encuentra compuesto por dos fases. La primera orientada a favorecer la comprensión y vivencia controlada de los diferentes elementos del marco de referencia explicado previamente, y la segunda, orientada a poner en acción los conocimientos a través de la realización de conversaciones con los sujetos apoderados.

Fase 1: Sensibilización y actualización. Esta fase tiene dos objetivos: el primero es analizar el comportamiento disruptivo de estudiantes a partir de la teoría del apego y su impacto en las actitudes y emociones del profesorado; el segundo es familiarizar y practicar, con el profesorado jefe, herramientas de control emocional que utilizará en las entrevistas con las personas apoderadas.

Para desarrollar estos objetivos se realizaron 8 sesiones, en las que se analizó bibliografía especializada, se discutieron casos propuestos por el equipo de formación y presentados posteriormente por el profesorado jefe, y se realizaron ejercicios físicos que permitieron reconocer el vínculo entre emoción, respiración y corporalidad. También, se desarrollaron actividades de escucha activa y argumentación a partir de evidencias de aprendizaje. Cada una de las personas participantes creó una bitácora de análisis de casos de sujetos apoderados y estudiantes de su jefatura de curso, la que fue compartida durante las sesiones. En esta fase se desarrollaron actividades que asociaban el problema, la emocionalidad, la disposición corporal del profesorado y el tipo de conversación que recordaban haber sostenido versus la que deberían sostener, acorde con los casos presentados.

Estas actividades teórico-prácticas permitieron al personal docente reconocer sus emociones y juicios sobre los sujetos apoderados y estudiantes, a partir de lo cual se establecieron las siguientes orientaciones para realizar las conversaciones desafiantes con los sujetos apoderados:

- Reconocer los estados emocionales a través de indicadores respiratorios, gestuales y subjetivos.

- Mantener la neutralidad emocional durante la conversación e implementar, de ser necesario, el step out.

- Más que decir, preguntar, con la finalidad de comprender el punto de vista del sujeto apoderado.

- Realizar preguntas abiertas, de tal forma que el sujeto apoderado pueda expresar completamente sus pensamientos.

- Escuchar activamente y asegurar la comprensión a través del parafraseo.

- Usar datos y evidencias de aprendizaje para nutrir la conversación y no rebatir sin elementos visuales que apoyen los puntos de vista. 
- Propiciar un rol activo de la persona apoderada, facilitando que él proponga caminos de acción y no imponiéndolos.

- Avanzar en pequeños pasos, potenciando que los compromisos sean logrables y evidenciables.

Aspectos administrativos:

- Cuidar la privacidad del lugar.

- Asegurarse de que ambas personas cuenten con tiempo suficiente.

- No realizar conversaciones de más de 30 minutos.

- Preparar las evidencias y llevarlas a la conversación.

- Resumir los acuerdos y consignar las estrategias de seguimiento de compromisos del sujeto apoderado y del profesorado.

Fase 2: Implementación de conversaciones desafiantes con sujetos apoderados. Esta segunda fase del proceso formativo tenía dos objetivos, el primero fue planificar, implementar y evaluar colaborativamente conversaciones desafiantes con los sujetos apoderados de estudiantes de bajo rendimiento o comportamiento disruptivo; el segundo, fortalecer la confianza profesional a través del trabajo colaborativo de duplas y el análisis de audios de las conversaciones realizadas.

Las conversaciones se planificaron de acuerdo con el modelo que está compuesto por 12 preguntas. La primera solicita realizar un resumen de la relación con el sujeto apoderado y declarar la situación que gatilla (quiebre) la citación. La segunda y tercera consultan sobre las disposiciones emocionales del profesorado jefe, respecto al estudiantado y el sujeto apoderado. En la cuarta se solicita realizar un análisis de las disposiciones emocionales declaradas en el punto anterior. En la quinta se sistematizan los datos e información que se utilizarán en las entrevistas. En la sexta se selecciona el tipo de conversación que se realizará. En la séptima y octava se solicita identificar el momento y lugar en que se realizará la entrevista. Las preguntas novena y décima solicitan analizar las disposiciones emocionales que no deben estar presentes y las que sí deben estar presentes en la entrevista. Finalmente, las preguntas decimoprimera y segunda abordan las metas de la conversación, de corto y largo plazo.

Seconformaron8duplas detrabajo,cadaunaplanificó,implementóyrecibióretroalimentación de dos conversaciones. En total se realizaron 18 conversaciones con sujetos apoderados, nueve corresponden al primer ciclo y las nueve restantes al segundo. Cada ciclo formativo se iniciaba con la planificación de la conversación, la que era retroalimentada por el equipo de personal formador. Posteriormente, cada dupla implementaba la conversación, la que era registrada en audio con la autorización del sujeto apoderado. Se solicitaba que la conversación fuera conducida por una sola persona docente y la segunda tuviera el rol de sombra. Luego, la dupla de docentes analizaba el 
audio utilizando la planificación realizada y la teoría estudiada. A continuación llegaba el turno del personal formador, quienes escuchaban el audio y retroalimentaban por escrito a cada dupla. En la sesión de clases, las duplas intercambiaban audios y planificaciones, y eran retroalimentadas por otra dupla de trabajo. Al final de la sesión, las duplas presentaban sus conclusiones. Esta secuencia de retroalimentación se realizó en el primer y segundo ciclo. Finalmente, cada dupla elaboró un escrito en que exponía y valoraba sus aprendizajes.

\section{Metodología}

El proceso formativo se evaluó utilizando el modelo de D. L. Kirkpatrick y J. D. Kirkpatrick (2006), que define cuatro niveles de evaluación: satisfacción, aprendizaje, comportamiento y resultados. Para evaluar el primer nivel se aplicó un cuestionario al final de la formación en que se midieron las siguientes dimensiones: calidad de docentes, pertinencia de la metodología, recursos y materiales utilizados y aspectos formales. Las opciones de respuesta iban desde muy de acuerdo (5) hasta muy en desacuerdo (1), y fueron analizadas utilizando estadísticos descriptivos. En el segundo nivel de evaluación, aprendizaje, se realizaron análisis de contenido de las planificaciones y audios de conversaciones. Para ello, se transcribieron los audios y se analizaron desarrollando categorías y códigos asociados al marco teórico desarrollado. El tercer nivel, comportamiento, se evaluó nueve meses después de finalizado el programa, a través de una encuesta dirigida a las personas participantes, que se analizó a través de estadísticos descriptivos y se profundizó a través de la técnica concept mapping (Johnson y Onweugbuzie, 2004), que consiste en la elaboración de mapas conceptuales por las personas participantes (Dagenais et al., 2012; Dagenais et al., 2015). A cada participante se le entregó un set de 10 tarjetas, en 5 de ellas debían identificar barreras para la implementación de la metodología de aprendizaje en el curso y en las otras 5 debían identificar elementos que favorecían su ejecución. Luego, se solicitó que jerarquizaran su importancia, utilizando un valor de 5 para la más importante y 1 para la menos importante. Una vez que cada una de las personas participantes jerarquizó sus tarjetas, se procedió a la organización en clúster. Para ello, se entregó a las personas participantes las siguientes orientaciones: un clúster es un grupo de aseveraciones que representan una misma perspectiva, que puede reunir en su interior aseveraciones de diferente nivel jerárquico y que requiere de un relato argumentativo de quienes participan. Esta técnica permite comprender los nodos e interrelaciones entre conceptos, con miras a descubrir patrones relacionales (Creswell, 2007). Estos mapas son útiles para acordar priorizaciones, establecer relación y evitar la repetición de ideas (Dagenais et al., 2015). El motivo por el cual se seleccionó esta técnica es porque permite identificar barreras y elementos favorecedores para continuar utilizando la metodología e identificar aspectos mejorables de la secuencia formativa implementada.

Finalmente, el cuarto nivel de evaluación, organizacional, tal como lo manifiestan Steensma y Groeneveld (2010), busca evaluar si los objetivos perseguidos por la organización al emprender la formación se cumplieron. Para ello se realizó un grupo de discusión que, según

10

Marta Quiroga-Lobos y Felipe Aravena-Castillo

Los artículos de la Revista Electrónica Educare del Centro de Investigación y Docencia en Educación de la Universidad Nacional, Costa Rica, se comparten bajo términos de la Licencia Creative Commons: Reconocimiento, № Comercial, Sin Obra Derivada 3.0 Costa Rica. Las autorizaciones adicionales a las aquí delimitadas se pueden obtener en el correo: educare@una.cr 
Stewart y Shamdasani (2014), permite conocer en profundidad e indagar en las opiniones de las personas participantes, en este caso del personal directivo docente, que como líderes de la organización perseguían determinados fines con el proceso formativo. Las preguntas esenciales que se trabajaron fueron: ¿Se lograron los objetivos del proceso formativo implementado? ¿Qué efecto tuvo el programa en la organización? ¿Se observaron cambios en la organización, y si fue así, de qué tipo?

La formación se implementó en una escuela básica que atiende a estudiantes entre 5 y 15 años, con una matrícula de 547 estudiantes, una planta docente de 42 y con un índice de vulnerabilidad del $87 \%$. El equipo directivo se encuentra compuesto por cuatro integrantes (director, jefe de UTP, inspector general y coordinador de convivencia escolar). Participaron en el curso 14 personas del profesorado jefe más el equipo directivo durante nueve meses; los tres primeros se destinaron al desarrollo de actividades conceptuales y en los seis siguientes se planificaron, implementaron y retroalimentaron las conversaciones realizadas con los sujetos apoderados. El promedio de edad fue de 38 años. De los participantes, 14 eran mujeres y 4 hombres, con una experiencia promedio como profesorado jefe de 10,7 años, y con 13,9 años promedio de experiencia docente.

\section{Resultados}

Los resultados son presentados en los cuatro niveles de evaluación.

a) Satisfacción: Una vez finalizado el curso, se aplicó una encuesta de satisfacción a las 18 personas participantes, que evaluó los siguientes aspectos: calidad del profesorado y materiales, metodología de clases, pertinencia de la evaluaciones y retroalimentación. Se construyó una encuesta con cinco opciones de respuesta, donde el valor 5 correspondía a "totalmente satisfecho/a"y 1 a "nada satisfecho/a". El $98 \%$ de los sujetos encuestados declaró estar totalmente de acuerdo con la metodología utilizada y la calidad docente. El 90 \% se mostró totalmente satisfecho con el sistema de evaluación y la retroalimentación entregada. Cuando se consultó si recomendarían el programa de formación a otros grupos de docentes, el $100 \%$ lo recomendaría.

b) Aprendizajes: Para esta fase se analizaron 18 planificaciones de conversaciones con los sujetos apoderados y sus respectivos audios de implementación. Primero se presentan los resultados de las planificaciones, en segundo lugar, el análisis de los audios y, finalmente, se integraron ambos. A continuación, el equipo formador solicitó a cada dupla seleccionar para esta experiencia formativa a sujetos apoderados con los que ya habían realizado entrevistas, y cuyos resultados no se habían ajustado a sus expectativas. A partir de estas indicaciones, cada dupla seleccionó a dos personas apoderadas, para la primera se planificó y ejecutó la conversación, lo que fue posteriormente 
retroalimentado por los pares y el equipo formador, con este conocimiento, cada dupla planificó e implementó una segunda conversación, poniendo en juego los conocimientos adquiridos en la primera entrevista.

La presentación de resultados de las planificaciones de entrevista se organizó de la siguiente forma: para las secciones desde la 1 a la 4, los datos se presentan agrupados, y desde la sección 5 en adelante, estos se presentan mostrando los cambios entre el primer y segundo ciclo, porque entre ambos se realiza la retroalimentación descrita en la sección del modelo formativo. A continuación, se presentan y discuten los resultados. De las 18 planificaciones de conversaciones, 13 corresponden a sujetos apoderados de estudiantes varones y 5 a alumnas mujeres. De los apoderados, solo uno es de género masculino (padre) y 17 de género femenino (4 abuelas por parte de madre y 13 madres de estudiantes).

En la primera sección de la planificación, se solicitó describir la relación con la persona apoderada. En síntesis, el profesorado jefe evaluó negativamente la experiencia, porque [refiriéndose a los sujetos apoderados] demuestran poco compromiso con sus hijos o nietos, o asumían compromisos en las conversaciones que luego no cumplían o tenían una actitud conflictiva con el profesor durante la conversación. A estas opiniones se agregan las descripciones de situaciones que gatillan la citación (quiebre), las que se agrupan de la siguiente forma: acusación del sujeto apoderado por accidente de su hijo o hija $(n=1)$, comportamiento agresivo del alumnado con docentes $(n=3)$, agresión física a compañeros o compañeras $(n=2)$, bajo rendimiento y ausentismo $(n=8)$, baja motivación en clases $(n=6)$ y comportamiento sexualizado de estudiantes con sus pares $(n=1)$.

Las aseveraciones del profesorado jefe, unidas a los eventos específicos que gatillan las entrevistas, se enmarcan en lo que Hargreaves (2001a) denomina distancia sociocultural entre padres y madres y profesorado, apoyada en la perspectiva del déficit (Goleman, 2006). El profesorado manifiesta que los padres y madres tienen poco que aportara los procesos formativos y que cuando se comprometen no son capaces de cumplir, lo que dificulta la construcción de relaciones respetuosas entre diferentes actores, ya que representan perspectivas socioculturales contrapuestas (Huss-Keeler, 1997; Olivos, 2006). Finalmente, es importante considerar que los discursos de docentes nacen de una perspectiva de la teoría de reproducción social y los de los padres y madres desde la perspectiva de las oportunidades que necesitan sus hijos e hijas para mejorar (Lawson, 2003).

En la segunda y tercera sección se solicitó al profesorado jefe declarar sus emociones sobre sus estudiantes, y padres y madres. En cuanto a estudiantes, las disposiciones emocionales se organizan de la siguiente forma: mezcla de pena y rabia $(n=4)$, porque docentes declaran comprender que el alumnado vive en condiciones muy difíciles, no obstante, sienten rabia porque las actitudes disruptivas en clases les impiden cumplir con su planificación y enseñar a sus demás estudiantes. Pena $(n=6)$, porque consideran que el contexto familiar en que vive

12 Marta Quiroga-Lobos y Felipe Aravena-Castillo

Los artículos de la Revista Electrónica Educare del Centro de Investigación y Docencia en Educación de la Universidad Nacional, Costa Rica, se comparten bajo términos de la Licencia Creative Commons: Reconocimiento, № Comercial, Sin 0bra Derivada 3.0 Costa Rica. Las autorizaciones adicionales a las aquí delimitadas se pueden obtener en el correo: educare@una.cr 
el alumnado lo perjudica para concentrarse en sus estudios, sienten que está desvalido. Rabia $(n=6)$, porque estudiantes se muestran desafiantes a las normas del establecimiento y a sus docentes. Ternura $(n=1)$, porque considera que para su estudiante, tener hermanos o hermanas de diferentes padres es una dificultad que impide desarrollar vínculos afectivos estables $y$, finalmente, un profesor jefe se declara en una emocionalidad neutral frente al estudiantado.

En cuanto a los padres y madres, la emoción de la rabia $(n=16)$ es la que predomina. El profesorado piensa que los sujetos apoderados están despreocupados por sus hijos, hijas o nietos y nietas, de acuerdo con las ideas expresadas en las planificaciones: sobrecargan a sus hijos o hijas mayores con el cuidado de hermanos o hermanas menores, los cambios constantes de relaciones de pareja de las madres provoca que su prole no tenga una figura paterna estable, falta de apoyo en el desarrollo de los hábitos, inasistencia a reuniones y citaciones, falta de hábitos familiares que inciden en el ausentismo escolar y, finalmente, sujetos apoderados muy exigentes en la búsqueda de beneficios sociales y poco cumplidores con sus deberes. Dos planificaciones declaran la emoción de pena, argumentan que se trata de personas apoderadas que no tienen la educación necesaria para apoyar a sus nietos o nietas, en estos casos la apoderada es la abuela materna de estudiantes.

La emoción de la rabia es la predominante en el profesorado jefe, por lo que es importante caracterizarla. Fisiológicamente por un patrón respiratorio, con ciclos rítmicos de alta frecuencia y de gran amplitud, donde predomina la respiración abdominal (Bloch, 2008). A nivel expresivo, los músculos faciales están en tensión, la mirada focalizada y el tono muscular va en aumento, el cuerpo y la cabeza se proyectan hacia delante. Subjetivamente la disposición mental está orientada a la extrema defensa o agresión. Todas las características fisiológicas, faciales, posturales y subjetivas enumeradas anteriormente son reconocibles universalmente, aunque en diferentes personas se manifiesten con más claridad que en otras. Las características de esta emoción y su predominancia, en las planificaciones de docentes, implica que la metodología CECA sea adecuada para lograr un control emocional durante las conversaciones, si se practican los ritmos de respiración del estado de neutralidad y se aplica la técnica de setp out. Ambas técnicas son fundamentales debido a la disposición negativa del profesorado jefe y personal directivo sobre estos sujetos apoderados, lo que les permitirá ampliar su comprensión, despejar gradualmente prejuicios y focalizar la conversación en los aprendizajes del alumnado.

La predominancia de la rabia como emoción en las planificaciones se asocia con la distancia sociocultural entre docentes y sujetos apoderados, y es uno de los desafíos importantes para el proceso formativo. Un primer paso dentro del guion de planificación, y uno muy relevante, es reconocer la emoción que les provocan estas personas apoderadas y comprender que esta emoción es fásica. Es decir, es episódica, de corta duración, y no marca la tonalidad o estado emocional del personal docente en un tiempo prolongado (Bloch, 2008); y que las técnicas de respiración, de neutralidad y step out son las que deben usar. La primera se caracteriza por un 
ritmo de respiración calmado y regular, que debe practicar antes de la entrevista y sostenerlo durante ella, lo que le permitirá escuchar, comprender y concentrando sus intervenciones en los aprendizajes del alumnado e ir abandonando, paulatinamente, la perspectiva del déficit. Indudablemente, en el transcurso de la entrevista los estados emocionales también están sujetos a cambios, por lo que es necesario que, una vez finalizada la conversación, cada docente aplique la técnica del step out, que puede ser caracterizada como un ejercicio. Esto le permite desprenderse de las emociones gatilladas en la conversación y retomar sus actividades de enseñanza, muy demandantes de control emocional.

Siguiendo el guion de planificación, las duplas debían seleccionar un tipo de conversación que se alineara al tipo de quiebre (situación que provocó la citación) y a la emoción declarada en el punto anterior de la planificación. Se seleccionaron tres tipos de conversaciones: juicio $(n=1)$, de coordinación para posibles acciones $(n=2)$ y de coordinación de acciones $(n=15)$. Estas conversaciones son adecuadas cuando subyacen acuerdos entre ambos sujetos hablantes, por lo que es muy probable llegar a acuerdos y realizar las acciones comprometidas.

Acorde con el análisis que el profesorado jefe realizó de su relación con las personas apoderadas y de la predominancia de la emoción de la rabia, la conversación para coordinar acciones suele no ser adecuada, ya que este tipo de conversaciones son factibles solo cuando hay acuerdos que subyacen entre las personas participantes de la conversación (Echeverría, 2007). Pareciera ser que cuando las duplas elaboran las planificaciones, reflejan una relación jerárquica y de poder hacia los sujetos apoderados, pues creen que así llegarán a acordar acciones. Esto cambia una vez que se realizan las retroalimentaciones, ya que la mayor parte de las conversaciones son reclasificadas como conversaciones para posibles acciones (Echeverría, 2007), tipo de conversación que permite al profesorado jefe escuchar y comprender profundamente las subjetividades de los sujetos apoderados y reconocer los fondos de conocimiento que ellos poseen y representan, en donde la coordinación de acciones es una posibilidad futura y no de corto plazo.

A continuación, se solicitaba identificar la información y datos que usarían en la entrevista. En las primeras nueve planificaciones, la información entregada se desprendía de la hoja de vida estudiantil, en forma de anotaciones de docentes, calificaciones y asistencia a clases, también se agregaba un relato de la situación específica que había gatillado la citación. En las siguientes nueve entrevistas, se solicitó al profesorado que en su planificación se incluyeran, junto a lo anterior, evidencias del desempeño estudiantil, ya fueran evaluaciones, guías de aprendizaje o actividades en clases, acompañadas de los criterios de evaluación, de tal forma que se favoreciera centrar la conversación en las brechas de aprendizaje y, a partir de esto, generar metas de corto plazo en las cuales el sujeto apoderado se pudiera comprometer. Este fue uno de los cambios que produjo más efecto en las dinámicas de las entrevistas ya que, al analizar los desempeños de sus hijos o hijas y las brechas, los sujetos apoderados se mostraron más activos 
al momento de proponer metas de corto plazo. Del seguimiento realizado por el profesorado jefe, se constató que los sujetos apoderados cumplían con las acciones comprometidas; no obstante, un número menor (3 personas apoderadas), dejó de cumplir con las acciones, con su consecuente decepción al no evidenciar cambios rápidos en sus hijos o hijas.

Una vez realizada la retroalimentación de las planificaciones de conversaciones, se iniciaba la implementación de las conversaciones con los sujetos apoderados. Éstas eran analizadas por la dupla y luego por el equipo formador. Estas grabaciones fueron transcritas y se analizaron de acuerdo con los contenidos trabajados en la primera etapa de la formación. A continuación, se analizan los resultados a partir de las transcripciones de estas conversaciones.

Al analizar los audios de las primeras nueve conversaciones se evidencia que contienen múltiple información y detalles de la vida hogareña del estudiantado con sus sujetos apoderados; se reconocen tensiones económicas, emocionales (respecto a otras personas de la familia, como los padrastros) y laborales, especialmente de las madres. Estos relatos se adueñan de las conversaciones, en gran medida, y el profesorado jefe participa activamente de ellas, ya sea mediante la formulación de preguntas abiertas, como por ejemplo: ¿Qué sientes tú cuando tu pareja dice eso? ¿Cómo has tratado de controlar esta situación?, entregando ejemplos de cómo ellos o ellas han resuelto problemas similares en su vida, por ejemplo: "A mi hija chica le pasaba lo mismo con su hermano [refiriéndose al hijo de su pareja], entonces yo los sacaba a pasear juntos para que aprendieran a compartir". O asumiendo una actitud más maternal con la apoderada: Tú aún eres muyjoven [le dice a la apoderada] para saber cómo seeduca a un hijo. Cuando se producen estos diálogos, el profesorado jefe aconseja a sus personas apoderadas sobre aspectos de su vida personal, demostrando que tienen experiencia y evidenciando, con ello, una distancia sociocultural (Hargreaves, 2001a) que permite aconsejarles en aspectos más íntimos de su vida, desvinculados del aprendizaje de sus proles. Por otra parte, el exceso de relatos de los sujetos apoderados sobre su vida personal y entorno familiar entrega nuevos antecedentes que fundamentan aún más la perspectiva del déficit y el hecho de que el profesorado jefe justifique los aprendizajes del estudiantado a partir de su entorno familiar y no, por ejemplo, a través de estrategias didácticas suyas y de sus colegas.

La dinámica de las conversaciones cambia cuando los sujetos apoderados reclaman sus derechos sociales, lo que queda reflejado en la siguiente conversación:

Apoderada: Yo ya conversé con usted [se refiere a la profesora] y le exijo que me entregue el computador que le corresponde a mi hijo.

Profesor jefe: Ya le expliqué que es solo para los mejores promedios y su hijo no está entre los mejores promedios del curso. 
Apoderada: Es que en las noticias del Mega [se refiere a un noticiero de televisión] no dicen eso, dicen que es para todos los niños de séptimo básico, entonces usted debe entregármelo. Yo voy a reclamar con el director ahora mismo y voy a hablar con el alcalde.

Profesor jefe: Usted es libre de hablar con quien quiera.

O cuando los sujetos apoderados plantean situaciones en que el profesorado es cuestionado:

Apoderada: Es que mi hijo le tiene medio a usted, porque dice que usted grita mucho y a él le da susto y por eso se pone a llorar.

Profesor jefe: Eso no es así, yo no grito en clases. Yo creo que usted tiene a su hijo muy regalón, por eso se asusta por todo y se pone a llorar.

En estas situaciones el profesorado siente cuestionada su autoridad, lo que, de acuerdo a Hargreaves (2001b), está asociado a una distancia moral, que no reconoce al sujeto apoderado la posibilidad de opinar sobre su trabajo o la información que entrega. En ambas entrevistas no se logra un acuerdo y el profesorado queda a merced de sentimientos de rabia.

En la segunda ronda de entrevistas, se solicitó a las duplas presentar al sujeto apoderado evidencias de aprendizaje del estudiantado y compartir los criterios de evaluación. Las siguientes citas reflejan opiniones de los sujetos apoderados: Yo nunca estudié esto en el colegio, no sabía qué hacía estas cosas en clases, es difícil esta pregunta, ¿otros niños la responden bien?, ¿qué significan estos cuadritos [refiriéndose a la rúbrica]?, ¿usted creer que la Martina logre aprender a desarrollar estos ejercicios? Las intervenciones de los sujetos apoderados se asocian a aspectos específicos de las tareas, al significado de los criterios de evaluación y a demandar al profesorado la seguridad de que su hijo o hija logrará los aprendizajes. Incluir estas evidencias en las entrevistas permitió desarrollar, junto con los sujetos apoderados, metas realistas y de corto plazo asociadas al aprendizaje. Por ejemplo: Yo podría leer todos los días un ratito, lo que usted [refiriéndose al profesor] marque en el libro de Lenguaje, y buscar con el Martín las palabras más difíciles. Se logró así una relación profesorado jefe-persona apoderada orientada al aprendizaje, en la que la persona apoderada participa de acuerdo con las necesidades de su hijo o hija y sus reales habilidades y disposiciones.

a) Comportamiento:Después de nueve meses de finalizado el curso, se aplicó una encuesta y se implementó la técnica concept mapping (creación de mapas conceptuales). Ambas tenían el objetivo de indagar en qué medida los conocimientos adquiridos en el curso se continuaban implementando e identificar elementos facilitadores y barreras. A continuación, se caracterizan y discuten los resultados de ambos instrumentos. 
La encuesta se estructuró en tres secciones, la primera evaluaba la permanencia de las prácticas de planificación de conversaciones, la segunda indagaba sobre las prácticas de implementación de conversaciones y la tercera sobre los procesos reflexivos asociados.

En relación con las prácticas de planificación, el 100 \% del profesorado jefe ha continuado planificandolas entrevistas, el 93,7\% analiza los prejuicios sobreel sujeto apoderado, el $82,7 \%$ revisa la historia relacional con el sujeto apoderado y la situación que gatilla la citación a entrevistas, el $87,2 \%$ identifica la emoción predominante con el sujeto apoderado y estudiante, el 62,5\% prepara y lleva a la entrevista evidencias del desempeño estudiantil, el $50 \%$ comparte la planificación con colegas, el $50 \%$ identifica el tipo de conversación y el $37 \%$ comparte su planificación con colegas. Como se observa, las prácticas de planificación se mantienen en el tiempo, especialmente en los aspectos de análisis de la relación con el sujeto apoderado y la emoción asociada. En cambio, los porcentajes asociados al trabajo colaborativo entre docentes son más bajos.

En la sección de la encuesta que consultaba sobre la implementación, se declaró que $100 \%$ logra reconocer sus estados emocionales antes, durante y después de la entrevista; $100 \%$ después de realizar las entrevistas tiene una sensación gratificante asociada al logro de objetivos y calidad de la conversación; $93,7 \%$ logra comprender el punto de vista de los sujetos apoderados y centrar las entrevistas en el aprendizajes de sus estudiantes, sin recabar o ahondar en información familiar; el 87,5 \% logra diseñar con sus sujetos apoderados metas de corto plazo; 81,5\% mantiene el estado de neutralidad durante las entrevistas, y $25 \%$ implementó la entrevista con colegas. Como se evidencia, los resultados son positivos, porque el profesorado jefe ha mantenido las orientaciones centrales del proceso formativo, logrando una relación positiva con los sujetos apoderados y finalizando las entrevistas con una sensación "gratificante" o de calma, por lo que no siempre necesitan realizar el ejercicio de step out. También, se observa que las prácticas de colaboración disminuyen drásticamente.

En la última sección, de reflexión, el 62,5 \% señaló que ha realizado un seguimiento de las metas de corto plazo comprometidas con el sujeto apoderado, el 43,75\% que compartió con colegas los resultados de la entrevista realizada, el 43,75 \% que solicitó colaboración a otras personas del profesorado del curso para identificar, en estudiantes, cambios asociados a las metas de corto plazo comprometidas, el 18,75\% que ha escuchado la grabación de la conversación y el $12,5 \%$ que ha escuchado y analizado las grabaciones con colegas. Tal como se evidencia en las otras secciones de la encuesta, existe una disminución de la colaboración entre pares, especialmente en esta última sección que potencia la comprensión del propio actuar y el desarrollo de estrategias compartidas, con el objetivo de mejorar la calidad de las conversaciones.

En la fase de comportamiento del modelo de evaluación también se aplicó la técnica de mapa conceptual, según los pasos descritos en la sección Metodología. Las personas participantes organizaron seis clústeres en donde agruparon los elementos facilitadores y cuatro agruparon las dificultades y barreras, para la implementación de la metodología CECA. 
Los elementos o factores facilitadores se organizaron en seis clústeres. El primero $(28,6$ $\%, \mathrm{n}=26$ ) reúne aseveraciones que destacan la importancia de contar con una metodología y vocabulario compartido entre el profesorado jefe; en el segundo $(18,9 \%, n=17)$, se considera que la metodología les ha permitido comprender más profundamente a sus sujetos apoderados; en el tercero $(16,7 \%, n=15)$, se considera que el compromiso con la jefatura de curso ha aumentado significativamente; en el cuarto $(12,2 \%, n=11)$, se ha ganado autonomía y seguridad para realizar conversaciones con los sujetos apoderados, lo que se expresa en pérdida de miedo a las personas apoderadas conflictivas; en el quinto $(12,2 \%, n=11)$, mejoran las habilidades de control emocional en las entrevistas con los sujetos apoderados y en resolución de conflictos con sus colegas, y en sexto lugar $(11,1 \%, n=10)$, destaca la importancia de que el equipo directivo participe en la formación. Finalmente, el profesorado jefe participante en la actividad decidió poner en el nodo central la siguiente aseveración: Una conversación desafiante es importante para el cambio.

Las dificultades o barreras para continuar implementando la metodología se agrupan en cinco clústeres. El primero se asocia al tiempo y espacio físico para realizar las entrevistas (32,2 \%, $n=2)$; el segundo $(22,2 \%, n=20)$ corresponde a las expectativas de los sujetos apoderados que esperan cambios rápidos y, si estos no se producen, se desaniman y abandonan los compromisos; el tercero $(16,7 \%, n=15)$ se asocia a las inasistencias de los sujetos apoderados a las citaciones; el cuarto $(14,4 \%, n=13)$ corresponde a la necesidad de seguir trabajando en el control emocional, especialmente en el estado de neutralidad en el desarrollo de las conversaciones; y el quinto $(14,4 \%, n=13)$ se asocia al desconocimiento de la metodología por otros u otras colegas.

El análisis de los clústeres entrega importantes pistas de cuáles son las fortalezas del modelo y las dificultades para continuar con la implementación, una vez finalizado el proceso formativo. Entre ellas se pueden agregar actividades que permitan al profesorado jefe diseñar estrategias para que los sujetos apoderados asistan a las conversaciones, analizar el significado que atribuyen a las inasistencias, cómo generar acciones institucionales que apoyen el proceso de citación y acciones de aseguramiento para lograr, efectivamente, la asistencia.

a) Organizacional: Se realizó un grupal focal con el personal directivo docente donde se discutieron tres temáticas: percepción del grado de cumplimiento de los objetivos de la formación, cambios organizacionales identificados y recomendaciones sobre la metodología CECA. La entrevista fue realizada por un profesional externo al equipo formador. Los datos fueron transcritos y luego codificados. Un primer aspecto que destacó fue la disminución de las entrevistas realizadas por el equipo directivo: Ahora tenemos más tiempo para nuestras actividades, cada vez atendemos menos apoderados. El profesorado jefe realiza la mayor parte de las entrevistas y solo solicita colaboración después de realizar varias entrevistas previas, lo que permitió solucionar uno de los problemas principales que gatillaron la asesoría. Un segundo aspecto que destacó fue el incremento de colaboración espontánea entre el profesorado jefe: Ellos (refiriéndose al profesorado jefe) se reúnen espontáneamente, están en la misma sintonía, comparten 
perspectivas sobre sus alumnos y apoderados. Una dificultad que ha enfrentado el equipo directivo es el cambio del personal del profesorado jefe, identifican que este es un grupo capacitado con vínculos profundos y que para el profesorado jefe nuevo es muy difícil comprender las dinámicas, el vocabulario e integrarse a las conversaciones. Esto también sucede con docentes que no pertenecen al profesorado jefe y se hace particularmente evidente en los consejos de docentes, cuando se analizan situaciones del alumnado. Finalmente, mencionan que las restricciones de tiempo han dificultado mantener el trabajo colaborativo de las duplas y entre ellas; adicionalmente, recomiendan que la formación debe ser entregada a todo el personal docente de la escuela, para poder contar con una base común en la implementación de conversaciones desafiantes con los sujetos apoderados.

\section{Conclusiones}

La evaluación de la metodología CECA, realizada a través del modelo de D. L. Kirkpatrick y J. D. Kirkpatrick (2006), entrega evidencia suficiente para identificar las potencialidades de la integración de las líneas bibliográficas señaladas y de las estrategias de formación implementadas, al lograr un impacto positivo en la organización escolar en donde se aplicó. Esto queda demostrado mediante la mantención de las prácticas y la solución a los problemas identificados por el equipo directivo que motivaron, en un primer lugar, la implementación de esta metodología. A continuación, se señalan las principales conclusiones que se desprenden, especialmente, de los resultados de las fases de comportamiento y organización del modelo de evaluación.

Un aspecto esencial de la metodología es el proceso de retroalimentación que se realiza entre pares y por personal experto, el que ayuda a modelar las interacciones verbales en las conversaciones, mantener el control emocional y conducir las conversaciones hacia el aprendizaje del alumnado. Esto potencia el establecimiento de vínculos de colaboración escuela-familia, que consideren las fortalezas de las familias y sus reales posibilidades contextuales. Con ello, el profesorado jefe ajusta sus expectativas al contexto sociocultural y emocional de las familias de sus estudiantes y abandona la suposición de que, provocando cambios primero en las familias, estos se reflejarán en los aprendizajes del alumnado. El profesorado jefe termina apoyando los procesos formativos de sus estudiantes con el foco en reducir las brechas de aprendizaje de su curso. Para ello, es fundamental que los equipo de gestión logren consolidar una visión compartida sobre el rol del profesorado jefe, considerando sus propósitos y resultados esperados.

La duración y frecuencia de la retroalimentación que necesita el profesorado jefe es un factor importante para el desarrollo de las habilidades conversacionales. Por ello se considera adecuado incorporar a la metodología una primera fase intensiva de retroalimentación y después realizar retroalimentaciones diferidas en el tiempo, focalizadas en temáticas 
específicas. Por otro lado, la metodología necesita incluir una formación diferenciada para el personal directivo docente, destinada a desarrollar conocimientos y habilidades para asegurar condiciones institucionales básicas que sostengan la implementación de la metodología. Estas son: a) gestión del tiempo institucional, organizar los horarios del profesorado para favorecer el trabajo colaborativo con foco en las necesidades de aprendizaje de sus estudiantes, b) gestión de infraestructura y espacios físicos, para implementar oficinas con la privacidad, silencio y mobiliario específico para realizar las conversaciones con los sujetos apoderados, y c) formar al profesorado en metodologías de animación de trabajo colaborativo y retroalimentación entre pares; apoyar el uso de las técnicas de control emocional; favorecer el análisis crítico de las conversaciones con sujetos apoderados y la mantención de un clima de respeto, apertura al aprendizaje y desarrollo profesional a través de las opiniones de colegas.

Finalmente, es necesario señalar que los programas de formación socioemocional destinados a mejorar la relación escuela-familia son aún escasos en nuestro país y en el contexto latinoamericano, y no necesariamente abordan la complejidad de la relación, pues ignoran, en gran medida, el tejido social y político de la emotividad (Dinallo, 2016), necesario de considerar especialmente en las escuelas que atienden alumnado vulnerable por la distancia sociocultural entre maestros, maestras, padres y madres. Las escuelas que han logrado mejorar su relación con las familias las han integrado como un actor activo en el proceso de mejora a través de instancias de participación auténticas. Un rol más activo y menos pasivo de las familias apoderadas requiere ser configurado en las escuelas chilenas de hoy.

\section{Referencias}

Anyon, J. (1980). Social class and the hidden curriculum of work. Journal of Education, 162(1), 6792. https://doi.org/10.1177/002205748016200106

U.S. Department of Education. (1994). Strong families, strong schools: Building community partnerships for learning. Autor. https://www.ncjrs.gov/pdffiles1/Digitization/154491NCJRS.pdf

Barnard, W. M. (2004). Parent involvement in elementary school and educational attainment. Children and Youth Services Review, 26(1), 39-62. https://doi.org/10.1016/j. childyouth.2003.11.002

Bergnehr, D. (2015). Advancing home-school relations through parent support? Ethnography and Education, 10(2), 170-184. https://doi.org/10.1080/17457823.2014.985240

Blase, J. y Anderson, G. (1995). The micropolitics of educational leadership: From control to empowerment. Cassell.

Bloch, S. (2008). Al alba de las emociones. Respiración y manejo de las emociones. Uqbar Editores.

Marta Quiroga-Lobos y Felipe Aravena-Castillo

Los artículos de la Revista Electrónica Educare del Centro de Investigación y Docencia en Educación de la Universidad Nacional, Costa Rica, se comparten bajo términos de la Licencia Creative Commons: Reconocimiento, № Comercial, Sin Obra Derivada 3.0 Costa Rica. Las autorizaciones adicionales a las aquí delimitadas se pueden obtener en el correo: educare@una.cr 
Bowlby, J. (1998). El apego y la pérdida (Vol 1). Paidós.

Bridges, M., Cohen, S. R. y Fuller, B. (2012). Abriendo puertas: Opening doors to opportunity. A national evaluation of second-generation trainers. University of California, Berkeley. Institute of Human Development.

Brunner, J. J. y Elacqua, G. (2003). Factores que inciden en una educación efectiva: Evidencia internacional. http://www.educoas.org/portal/bdigital/lae-ducacion/139/pdfs/139pdf1.pdf

de Carvalho, M. E. P. (2001). Rethinking family-school relations: A critique of parental involving in schooling. Erlbaum Associates. https://doi.org/10.4324/9781410600332

Casanova, U. (1996). Parent involvement: A call for prudence. Educational Researcher, 25(8), 3046. https://doi.org/10.3102/0013189X025008030

Casassus, J. (Coord.), Cusato, S. Froemel, J. E. y Palafox, J. C. (Consultores). (2001). Primer estudio internacional comparativo sobre lenguaje, matemática y factores asociados, para alumnos de tercer y cuarto grado de la educación básica. Informe técnico. Laboratorio Latinoamericano de Evaluación de la Calidad de la Educación, UNESCO. https://unesdoc.unesco.org/ ark:/48223/pf0000149268

CIDE y UNICEF. (2000). Diálogo social: Participación de los centros de padres en la educación. Autor.

Clandinin, D. J. y Connelly, F. M. (1992). Teacher as curriculum maker. En P. W. Jackson (Ed.), Handbook of research on curriculum (pp. 363-401). Macmillan.

Clandinin, D. J., Huber, J., Huber, M., Murphy, M. S., Murray, A., Pearce, M. y Steeves, P. (2006). Composing diverse identities: Narrative inquiries into the interwoven lives of children and teachers. Routledge. https://doi.org/10.4324/9780203012468

Clunies-Ross, P., Litlle, E y Kienhuis, M. (2008). Self-reported and actual use of proactive and reactive classroom management strategies and their relationship with teacher stress and student behaviour. Journal of Educational Psychology, 28(6), 693-710. https://doi. org/10.1080/01443410802206700

Collao, O., Irrazabal, R. y Oyarzún, A. (1998). Un nuevo espacio, nuevas imágenes juveniles. Los consejos de curso: Percepciones, valoraciones y expectativas. Ediciones CIDPA.

Creswell, J. W. (2007). Qualitative inquiry \& research design: Choosing among five approaches (2. ${ }^{\text {a }}$ ed.). Sage.

Dagenais, C., Pinard, R., St-Pierre, M., Briand-Lamarche, M., Cantave, A. K. y Péladeau, N. (2015). Using concept mapping to identify conditions that foster knowledge translation from the perspective of school practitioners. Research Evaluation, 25(1), 70-78. https://doi. org/10.1093/reseval/rvv026 
Dagenais, C., Ridde, V. y Péladeau, N. (Octubre, 2012). The concept mapping methodology: A review of users'Evaluative comments. En 26th Annual Conference of the American Evaluation Association. Minneapolis, Minnesota.

Delpit, L. (1993). Other people's children: Cultural conflict in the classroom. The New Press.

Dinallo, A. M. (2016). Social and emotional learning with families. Journal of Education and Learning, 5(4), 147-158. https://doi.org/10.5539/jel.v5n4p147

Domina, T. (2005). Leveling the home advantage: Assessing the effectiveness of parental involvement in elementary school. Sociology of Education, 78(3), 233-249. https://doi. org/10.1177/003804070507800303

Dorthe Tveit, A. (2009) A parental voice: Parents as equal and dependent - rhetoric about parents, teachers, and their conversations. Educational Review, 61(3), 289-300. https://doi. org/10.1080/00131910903045930

Echeverría, R. (2007). Actos de lenguaje. Vol 1: La Escucha. Granica Adelphi.

Epstein, J. L. (2001). School, family, and community partnerships: Preparing educators and improving schools. Westview Press.

Eurydice. (1997). The role of the parents in the education systems of the European Union. Brussels: The Information network on Education in Europe. http://www.sel-gipes.com/ uploads/1/2/3/3/12332890/1997 eurydice - the role of parents in the education systems of the european union.pdf

Fan, W. y Williams, C. M. (2010). The effects of parental involvement on students' academic self-efficacy, engagement and intrinsic motivation. Educational Psychology, 30(1), 53-74. https://doi.org/10.1080/01443410903353302

Fonagy, P. Gergely, G. Jurist, E. L. y Target, M. (2002). Affect regulation, mentalization, and the development of the self. Other Press.

Geddes, H. (2006). El apego en el aula: Relación entre las primeras experiencias infantiles, el bienestar emocional y el rendimiento escolar. Graó.

Goleman, D. (2006). Emotional intelligence: Why it can matter more than IQ. Bantam.

Grumet, M. (1988). Bitter milk: Women and teaching. University of Massachusetts Press.

Hargreaves, A. (2001a). Emotional geographies of teaching. Teachers College Record, 103(6), 1056 - 1080. https://doi.org/10.1111/0161-4681.00142 
Hargreaves, A. (2001b). The emotional geographies of teachers' relations with colleagues. International Journal of Educational Research, 35(5), 503-527. https://doi.org/10.1016/ $\underline{\text { S0883-0355(02)00006-X }}$

Hargreaves, A. y Goodson, I. (1996). Teachers' professional lives: Aspirations and actualities. En I. F. Goodson y A. Hargreaves (Eds.), Teachers' professional lives (pp. 1-28). Falmer Press.

Henderson, A. T., Mapp, K. L, Johnson, V. R. y Davies, D. (2007). Beyond the bake sale: The essential guide to family-school partnerships. The New Press.

Hughes, N. K. y Schlösser, A. (2014). The effectiveness of nurture groups: A systematic review. Emotional and Behavioural Difficulties, 19(4), 386-409. https://doi.org/10.1080/13632752. 2014.883729

Huss-Keeler, R.L.(1997).Teacher perception of ethnicand linguisticminority parental involvement and its relationship to children's language and literacy learning: A case study. Teacher and Teacher Education, 13(2), 171-182. https://doi.org/10.1016/S0742-051X(96)00018-2

Jeynes, W. H. (2005). A meta-analysis of the relation of parental involvement to urban elementary school student academic achievement. Urban Education, 40(3), 237-269. https://doi. org/10.1177/0042085905274540

Jeynes, W. (2012). A meta-analysis of the efficacy of different types of parental involvement programs for urban students. Urban Education, 47(4), 706-742. https://doi. org/10.1177/0042085912445643

Johnson, B. R. y Onwuegbuzie, A. J. (2004). Mixed methods research: A research paradigm whose time has come. Educational Researcher, 33(7), 14-26. https://doi. org/10.3102/0013189X033007014

Kirkpatrick, D. L. y Kirkpatrick, J. D. (2006). Evaluating training programs: The four levels. BerrettKoehler Publishers.

Lasky, S. (2000). The cultural and emotional politics of teacher-parent interactions. Teaching and Teacher Education, 16(8), 843-860. https://doi.org/10.1016/S0742-051X(00)00030-5

Lawson, M. A. (2003). School-family relations in context parent and teacher perceptions of parent involvement. Urban Education, 38(1), 77-133. https://doi.org/10.1177/0042085902238687

Le Fevre, D. M. y Robinson, V. M. (2014). The interpersonal challenges of instructional leadership: Principals' effectiveness in conversations about performance issues. Educational Administration Quarterly, 51(1), 58-95. https://doi.org/10.1177/0013161X13518218 
Lindblad, S. y Pérez Prieto, H. (1992). School experiences ad teacher socialization: A longitudinal study of pupils who grew up to be teachers. Teaching and Teacher Education, 8(5-6), 465470. https://doi.org/10.1016/0742-051X(92)90051-4

Marchant Orrego, T., Milicic Muller, N. y Álamos Valenzuela, P. (2013). Impacto en los niños de un programa de desarrollo socio-emocional en dos colegios vulnerables en Chile. Revista Iberoamericana de Evaluación Educativa, 6(2), 167-186. https://revistas.uam.es/index.php/ riee/article/view/3411/3628

Martiniello, M. (1999). Participación de los padres en la educación: Hacia una taxonomía para América Latina. En Development Discussion Paper No. 709. Harvard Institute for International Development. http://x.incae.edu/ES/clacds/publicaciones/articulos/cen1202.php

Marrone, M. (2001). La teoría del apego. Un enfoque actual. Editorial Psimática.

Olivos, E. M. (2006). The power of parents: A critical perspective of bicultural parent involvement in public schools. Peter Lang.

Pinedo Palacios, J. R. y Santelices Álvarez, M. P. (2006). Apego adulto: Los modelos operantes internos y la teoría de la mente. Terapia Psicológica, 24(2), 201-210. http://www.redalyc. org/articulo.oa?id=78524210

Powell-Smith, K. A., Shinn, M. R., Stoner, G., y Good, R. H, III. (2000). Parent tutoring in reading using literature and curriculum materials: Impact on student reading achievement. School Psychology Review, 29(1), 5-27.

Pushor, D. (2007). Welcoming parents: Educators as guest hosts on school landscapes. Education Canada, 47(4), 6-11. https://www.edcan.ca/articles/welcoming-parents-educators-asguest-hosts-on-school-landscapes/

Ramírez, A. Y. F. (2003). Dismay and disappointment: Parental involvement of latino immigrant parents. The Urban Review, 35(2), 93-110. https://doi.org/10.1023/A:1023705511946

Ramírez Muga, M. Á. (2015). La labor del/la profesor/a jefe/a y su des-politización. Revista Iberoamericana de Producción Académica y Gestión Educativa, 2(4), 12-24. http://www.pag. org.mx/index.php/PAG/article/view/295/339

Sheldon, S. B. y Epstein, J. L. (2005). Involvement counts: Family and community partnerships and mathematics achievement. The Journal of Educational Research, 98(4), 196-206. https:// doi.org/10.3200/JOER.98.4.196-207

Simon, B. S. (2001). Family involvement in high school: Predictors and effects. NASSP Bulletin, 85(627), 8-19. https://doi.org/10.1177/019263650108562702 
Sirvani, H. (2007). The effect of teacher communication with parents on students' mathematics achievement. American Secondary Education, 36(1), 31-46.

Steensma, H. y Groeneveld, K. (2010). Evaluating a training using the "four levels model". Journal of Workplace Learning, 22(5), 319-331. https://doi.org/10.1108/13665621011053226

Stewart, D. W. y Shamdasani, P. N. (2014). Focus groups: Theory and practice. Sage.

Villarroel, V. A. (2011). Relación entre autoconcepto y rendimiento académico. Psykhe, 10(1), 3-18. http://www.psykhe.cl/index.php/psykhe/article/view/418/398

Waggoner, K., y Griffith, A. (1998). Parent involvement in education. Ideology and experience. Journal for a Just and Caring Education, 4(1), 65-77.

Wong, H., Wong, R., Rogers, K., y Brooks, A. (2012). Managing your classroom for success. Science and Children, 49(9), 60-64.

Yosso, T. J. (2005). Whose culture has capital? A critical race theory discussion of community cultural wealth. Race Ethnicity and Education, 8(1), 69-91. https://doi. org/10.1080/1361332052000341006 